\title{
DINÂMICA POPULACIONAL DE BOSTRICHIDAE, PLATYPODIDAE E SCOLYTIDAE (COLEOPTERA) EM TALHÃO DE UROGRANDIS (Eucalyptus urophylla $x$ Eucalyptus grandis) NO MUNICÍPIO DE CUIABÁ, ESTADO DE MATO GROSSO
}

\author{
José Renato Maurício da Rocha ${ }^{1}$; Alberto Dorval ${ }^{2}$; Otávio Peres Filho ${ }^{2}$; Reginaldo Brito da \\ Costa $^{2}$
}

${ }^{1}$ Engenheiro Florestal, Mestrando do PPGCFA/FENF/UFMT, Rua Bento A. dos Santos, 717, Centro, Mirassol
D’Oeste-MT, CEP: 78280-000, E-mail: jrm_rocha@ hotmail.com
${ }^{2}$ Faculdade de Engenharia Florestal FENF / UFMT. Av. Fernando Correa da Costa, no 2367 - Bairro Boa Esperança, Cuiabá-MT
CEP: 78060-900 E-mail: a.dorval@ hotmail.com; peres@ @ufmt.br; reg.brito.costa@ gmail.com

\section{RESUMO}

O objetivo desta pesquisa foi qualificar e quantificar as populações de Bostrichidae, Platypodidae e Scolytidae que ocorrem associadas ao híbrido Urograndis (Eucalyptus urophylla $\mathrm{x}$ Eucalyptus grandis) nos períodos de seca (maio a outubro) e de chuva (novembro a abril), no município de Cuiabá, estado de Mato Grosso, Brasil. As coletas foram quinzenais e ocorreram de março de 2008 a fevereiro de 2009. Foram utilizadas seis armadilhas etanólicas iscada com álcool combustível hidratado. As armadilhas foram distribuídas em duas linhas, sendo três por linha, com distância de 30m entre linhas e armadilhas. Os exemplares coletados foram triados, contados e identificados nos Laboratórios de Proteção Florestal da Universidade Federal de Mato Grosso e da Universidade Federal do Paraná. Foram realizados estudos faunísticos de dominância, constância, frequiência, abundância e de diversidade. Também foi desenvolvido estudo de flutuação populacional, de correlação com a temperatura, umidade relativa, precipitação pluvial e testes de médias entre as espécies coletadas. Foram identificadas 28 espécies e 997 indivíduos, em ambos os períodos analisados. Do total de indivíduos coletados, 642 ocorreram no período de seca e 350 no período de chuva. Scolytidade com $81,24 \%$ dos indivídus coletados em ambos os períodos, foi a família mais representativa. Premnobius cavipennis e Xyleborus affinis foram as espécies mais numerosas, independente do período analisado. As espécies Bostrichopsis uncinata e Xyleborus spinulosus ocorreram como dominante, muito abundante, muito freqüente e constante somente no período seco, enquanto que no período de chuvas, Premnobius cavipennis, Xyleborus affinis e Xyleborus ferrugineus ocorreram como super dominante, super abundante, super freqüente e constante. No período de seca, os índices de Eqüitatibilidade e de ShannonWiener foram mais expressivos quando comparados com os obtidos nos meses de chuva. Todas as espécies ocorreram com picos populacionais nos meses do período seco.

Palavras-chave: Coleoptera, coleobrocas, eucalipto híbrido, Eucalyptus 


\section{POPULATION DYNAMICS OF BOSTRICHIDAE, PLATYPODIDAE AND SCOLYTIDAE (COLEOPTERA) IN TALHÃO OF UROGRANDIS (Eucalyptus grandis $\mathbf{x}$ Eucalyptus urophylla) IN THE CITY OF CUIABÁ, STATE OF MATO GROSSO}

\section{ABSTRACT}

The purpose of this research was to qualify and quantify populations of Bostrichidae, Platypodidae and Scolytidae associated to an Urograndis hybrid stand (Eucalyptus urophylla $\mathrm{x}$ Eucalyptus grandis) during dry (May to October) and rainy (November to April) seasons in Cuiabá, State of Mato Grosso, Brazil. Collectings occurred every 15 days from March 2008 to February 2009. Six ethanolic traps were used. Traps were distributed in two rows, three per row, $30 \mathrm{~m}$ apart from rows and traps. The following indexes were estimated: faunal dominance, constancy, frequency, abundance and diversity. Population fluctuation studies were also conducted and correlation tests analysing temperature, relative humidity, rainfall. Means were compared to collected species and season periods. Twenty-eigth species were identified among 997 specimens. Among the collected specimens, 642 were obtained during the dry season and 350 during the rainy season. Totalizing $81.24 \%$ of the collected specimens, Scolytidae was the most representative family in the periods analized. Premnobius cavipennis and Xyleborus affinis were the most abundant species, regardless the weather period. Bostrichopsis uncinata and Xyleborus spinulosus were dominant, very abundant, very frequent and constant only during dry season, whereas in the wet season Premnobius cavipennis, Xyleborus affinis and Xyleborus ferrugineus were extremely dominant, extremely abundant, extremely frequent and constant. During the dry season, the Equitatibility and Shannon-Wiener indexes were more expressives when compared to the ones of the rainy months. All species presented population peaks in the dry months.

Key words: Coleoptera, Woodborers, hybrid eucalypt, Eucalyptus

\section{INTRODUÇÃO}

O melhoramento genético no gênero Eucalyptus tem fornecido novos clones com características favoráveis, como resistência às pragas, doenças e aumento no incremento volumétrico de madeira. Entretanto, alguns clones plantados em várias regiões do estado de Mato Grosso não estão adaptados às condições climáticas e edáficas da região, pois foram desenvolvidos para condições ambientais das regiões Sul e Sudeste do Brasil.

Além da incompatibilidade entre procedência e o destino dos híbridos plantados, diversas espécies de insetos que ocorrem associados aos plantios de Eucalyptus spp. tem potencial para se constituírem pragas a alguns dos clones de eucaliptos plantados na região Centro-Oeste, principalmente, no estado de Mato grosso. $\mathrm{O}$ híbrido Urograndis é atualmente o mais utilizado pelas empresas reflorestadoras no estado de Mato Grosso em plantios destinados à produção de lenha. De acordo com Trefflich (2003) os híbridos de Eucalytpus urophylla x Eucalyptus grandis são de grande aceitação por empresas produtoras de celulose no estado do Espírito Santo, por sua boa produtividade e resistência à formação de cancros.

$\mathrm{O}$ estudo da diversidade destes grupos de insetos tem abordado o comportamento das espécies em ambientes naturais e reflorestados, permitindo desta forma identificar as espécies mais ou menos 
predominantes nestes ambientes. Os coleópteros representam atualmente o terceiro grupo de insetos mais importantes para a silvicultura brasileira, principalmente as espécies das famílias Buprestidae, Cerambycidae, Chrysomelidae, Curculionidae, Platypodidae, Scolytidae e Scarabaeidae (Pedrosa Macedo, 1993; Zanuncio et al., 1993; Berti Filho, 1979; 1981). Outra família importante para o setor florestal é Bostrichidae, que compreende cerca de 90 gêneros e 700 espécies com distribuição tropical, sendo que no Brasil ocorrem aproximadamente 15 gêneros e 34 espécies (Matoski, 2005) e são adaptados à xilofagia, com as larvas e adultos alimentando-se dos tecidos de plantas lenhosas. Algumas espécies são economicamente importantes, como Sinoxylon conigerum, Rhyzopertha dominica, Prostephanus truncatus, Sinoxylon spp., Dinoderus spp., Lyctus spp. (Liu et al., 2008; Peres Filho et al., 2006).

As espécies das famílias Scolytidae e Platypodidae são comuns em áreas reflorestadas e muitas são consideradas pragas de importância secundária, colonizando hospedeiros fragilizados por doenças, estressados, galhos e ramos oriundos da desrama natural ou artificial dentro de áreas reflorestadas. Entretanto, nestas famílias, existem espécies que atacam madeiras recém cortadas e estocadas a beira dos talhões e nos pátios de armazenagem aguardando seu beneficiamento causando danos consideráveis.

O hábito de escavar galerias para cultivar seu fungo simbionte, causa manchamento e depreciação do valor comercial da madeira (Dorval, 2002).

As espécies Platypus papallelus, Platypus sp. (Platypodidae), Xyleborus ferrugineus, Xyleborus affinis, Xyleborus volvulus (Scolytidae) foram coletados em madeira de 13 espécies florestais nativas em madeireiras de Manaus por Abreu et. al. (2002). Dorval et. al. (2004) registraram pela primeira vez, no município de Cuiabá/MT, os ataques de Playtpus linearis, Platypus sp. (Platypodidae), Hypothenemus eruditus, X. affinis, X. ferrugineus e de Cryptocarenus heveae em madeiras com casca estocadas por um período de 30 a 180 dias de Eucalyptus camaldulensis, Eucalyptus citriodora, Eucalyptus urophylla e de Eucalyptus pellita concluindo que madeiras de E. camaldulensis, indepedente do período de estocagem, são mais resistentes, exibindo menor atratividade aos insetos.

Este estudo teve como objetivo investigar a diversidade de espécies de Bostrichidae, Platypodidae e Scolytidae que ocorrem associados a um plantio de urograndis (Eucalyptus urophylla $\mathrm{x}$ Eucalyptus grandis) nos períodos de seca e de chuva, no município de Cuiabá, estado de Mato Grosso.

\section{MATERIAL E MÉTODOS}

O estudo foi realizado de Abril de 2008 a Março de 2009 na fazenda Mutuca, localizada no município de Cuiabá-MT, (coordenadas geográficas $15^{\circ} 18^{\prime} \mathrm{S}$ e 55 $59^{\prime} \mathrm{W}$ da sede da fazenda). As armadilhas foram instaladas em um talhão de 3,83 ha do híbrido urograndis (Eucalyptus urophylla $\mathrm{x}$ Eucalyptus grandis) com 3,5 anos de idade.

O relevo da área varia entre formação plana e suavemente ondulada, com altitude média de 800 metros acima do nível do mar. O solo da região é do tipo areia quartzosa álica, de textura arenosa, destituído de minerais primários, pouco resistente ao intemperismo e de baixa fertilidade. A composição da vegetação natural da região é do tipo cerrado ralo, campos, matas ciliares e cerradão (Conceição, 1997). O clima da região é classificado como "Aw" (Köppen), com temperatura média anual de $26^{\circ} \mathrm{C}$ e precipitação pluviométrica média anual de $1400 \mathrm{~mm}$.

Neste levantamento foram utilizadas seis armadilhas etanólicas modelo 
escolitídeo/Curitiba modificada, dispostas em duas linhas contendo três armadilhas por linha. A distância foi de $30 \mathrm{~m}$ entre armadilhas e linhas e a 50 metros das margens externas dos talhões para evitar o efeito de borda. As armadilhas foram instaladas a $1,5 \mathrm{~m}$ da superfície do solo em relação à borda do funil coletor. Após cada coleta, as armadilhas foram abastecidas com etanol combustível $93 \%$ como atrativo e com etanol $70 \%$ no frasco coletor.

As coletas ocorreram quinzenalmente nos períodos de seca (maio a outubro) e de chuva (novembro a abril) e os espécimes coletados foram acondicionados em recipientes apropriados, identificados com o número da armadilha e data de coleta. Posteriormente, foram transportados para o Laboratório de Proteção Florestal da Universidade Federal de Mato Grosso, onde foram triados e secos em estufa a $60^{\circ} \mathrm{C}$ por 72 horas. Após esta fase, os exemplares receberam códigos e foram armazenados. Os espécimes coletados foram identificados taxonomicamente por comparação com material entomológico depositado nas coleções dos Laboratórios de Proteção Florestal, das Faculdades de Engenharia Florestal, da Universidade Federal de Mato Grosso e Universidade Federal do Paraná. Também foram enviados exemplares de Scolytidae ao Prof. Dr. Eli Nunes Marques e de Bostrichidiae ao Dr. Edson Possidônio Teixeira para identificação.

As análises, qualitativa e quantitativa, para os períodos de seca (maio a outubro) e de chuva (novembro a abril) foram feitas mediante contagem direta dos indivíduos e os cálculos da frequiência, constância, dominância, abundância e diversidade para cada período foram feitos utilizando o software ANAFAU (Moraes \& Silveira Neto, 2003). As médias das coletas de cada espécie nos perídos de seca e de chuva foram comparadas pelo teste de ScottKnot a 5\% de probabilidade. Também foram realizados estudos de flutuação populacional e testes de correlação de Pearson ao nível de significância de $1 \%$ e $5 \%$ de probabilidade entre as temperaturas máxima, média e mínima, com a umidade relativa e precipitação pluvial e as espécies que ocorreram na análise faunística anual como dominantes, muito abundantes, muito freqüentes e constantes.

Os valores originais das coletas foram transformados pela equação $\log (\mathrm{x}+1)$ para efeito de cálculos estatísticos e também foram submetidos aos testes de normalidade de Lilliefors (Campos, 1976) e de homogeneidade de variância de Cochran (Costa Neto, 1977). Foi utilizado o delineamento inteiramente casualizado em esquema fatorial $25 \times 2$ (25 espécies por 2 períodos (seca e chuva) com seis armadilhas/repetições, constituindo 300 parcelas.

\section{RESULTADOS E DISCUSSÃO}

No talhão de urograndis foram coletadas 28 espécies, distribuídas em Bostrichidae com quatro, Platypodidae com duas e Scolytidae com 22 espécies. A família Scolytidae foi a mais diversificada, ocorrendo nos períodos de seca e de chuva com $16(69,57 \%)$ e $19(76 \%)$ espécies, respectivamente (Tabela 1). Nos meses de seca coletou-se 496 indivíduos, o que corresponde a $67,72 \%$ dos espécimes em ambos os períodos analisados.

A família Scolytidae foi a mais representativa em quantidade de espécimes coletados (Tabela 1).

Dorval et. al. (2004) coletaram em talhão de E. urophylla, no município de Cuiabá/MT, 19 espécies e 3.165 espécimes da família Scolytidae no período seco. Trinta espécies e 3.372 indivíduos foram coletados no período de chuva, observando que $C$. diadematus, $C$. heveae, $C$. seriatus, $H$. obscurus e $X$ spinosulus ocorrem em elevada densidade populacional, independente do período de seca e de chuva. 
Tabela 1. Número de espécies indivíduos coletados com armadilhas etanólicas nos períodos seco e de chuva em talhão de urograndis (Eucalyptus urophylla x Eucalyptus grandis) no município de Cuiabá-MT.

\begin{tabular}{lcccccccc}
\hline \multirow{2}{*}{ Família } & \multicolumn{9}{c}{ Períodos } \\
\cline { 2 - 9 } & \multicolumn{9}{c}{ Seco } & \multicolumn{5}{c}{ Chuva } \\
\cline { 2 - 9 } & $\mathrm{E}$ & $\%$ & $\mathrm{I}$ & $\%$ & $\mathrm{E}$ & $\%$ & $\mathrm{I}$ & $\%$ \\
\hline Bostrichidae & 5 & 21,7 & 106 & 16,5 & 4 & 16,0 & 23 & 6,6 \\
Platypodidae & 2 & 8,7 & 40 & 6,2 & 2 & 8,0 & 13 & 3,7 \\
Scolytidae & 16 & 69,6 & 496 & 77,3 & 19 & 76,0 & 314 & 89,7 \\
\hline Total & 23 & 100 & 642 & 100 & 25 & 100 & 350 & 100 \\
\hline
\end{tabular}

E: Espécies; I: Indivíduos.

Corroborando estas informações Müller \& Andreiv (2004) observaram em plantios de E. grandis, no município de Blumenau/SC 21 espécies de Scolytidae, sendo $X$. affinis, $H$. eruditus e $X$. gracillis as mais coletadas e constantes.

Observa-se que em Bostrichidae, $16,51 \%$ dos indivíduos coletados ocorreram nos meses de seca e que B. uncinata e Micrapate sp. foram as espécies mais abundantes (Tabela 2).

A família Bostrichidae correspondeu a $16,51 \%$ dos espécimes coletados no período de seca. Neste ambiente, 20 espécies ocorreram indistintamente nos dois períodos. No período seco, B. uncinata (Bostrichidae), $X$. squamulatus e $X$. spinulosus (Scolytidae) foram as espécies mais abundantes, enquanto que $P$. cavipennis e $X$. affinis exibiram também alta abundância, porém independente do período analisado (Tabela 2).

As espécies Cocotrypes sp., $X$. compactus, $X$. neivai, $X$. squamulatus e Tricolus sp. (Scolytidae) foram coletadas somente no período de chuva, enquanto que C. convexicauda, $H$. bolivianus e $X$. hagedornii (Scolytidae) somente foram encontradas nos meses do período seco (Tabela 2).

A ocorrência de $P$. cavipennis com altas densidades populacionais em áreas reflorestadas com Eucalyptus spp. é considerada comum, pois a espécie é importante praga florestal. Isto é confirmado por Santos et al. (2003) que observaram a ocorrência de $P$. cavipennis em plantios de E. urophylla, no município de Niquelândia/GO, com percentual de 50,29.

Peres Filho et al. (2006) observaram em pátio de serraria, no município de Sinop/MT, dominância de $P$. linearis, $X$. affinis, X. picea, Platypus sp., H. eruditus e Xyleborus sp.

Oliveira et al. (2001) coletaram com armadilhas luminosas, indivíduos de $B$. uncinata, espécie considerada bastante agressiva à madeira de várias espécies de eucalipto e de Xyleborus volvulus em talhões de $E$. grandis no município de Nova Era/MG, indicando que algumas espécies de coleobrocas encontram-se bem adaptadas a ambientes reflorestados.

No talhão de urograndis, 11 espécies dominantes, quatro muito abundantes, cinco muito freqüentes e 16 constantes foram registradas na análise anual. No período seco, nove foram dominantes, cinco muito abundantes e freqüentes e 17 constantes. Já nos meses de chuva, sete foram dominantes, três muito abundantes, cinco muito freqüentes e 12 constantes (Tabela 3 ). 
Dinâmica populacional de Bostrichidae, Platypodidae e Scolytidae (Coleoptera) em talhão de urograndis (Eucalyptus urophylla $x$ Eucalyptus grandis) no município de Cuiabá, estado de Mato Grosso

Tabela 2. Abundância de espécies classificadas por família em coletas realizadas com armadilhas etanólicas nos períodos de seca e chuva em talhão de urograndis (Eucalyptus urophylla x Eucalyptus grandis), município de Cuiabá-MT.

\begin{tabular}{|c|c|c|c|c|c|c|}
\hline \multirow{3}{*}{ Família/Espécie } & \multicolumn{6}{|c|}{ Período } \\
\hline & \multicolumn{2}{|c|}{ Seco } & \multicolumn{2}{|c|}{ Chuva } & \multicolumn{2}{|c|}{ Anual } \\
\hline & Ind. & $\%$ & Ind. & $\%$ & Ind. & $\%$ \\
\hline \multicolumn{7}{|l|}{ Bostrichidae } \\
\hline Bostrychopsis uncinata & 53 & 8,26 & 5 & 1,43 & 58 & 5,85 \\
\hline Micrapate $\mathrm{sp}$ & 31 & 4,83 & 5 & 1,43 & 36 & 3,68 \\
\hline Xyloperthela picea & 16 & 2,49 & 2 & 0,57 & 18 & 1,81 \\
\hline Xyloprista sp. & 6 & 0,93 & 11 & 3,14 & 17 & 1,71 \\
\hline Sub-total (Bostrichidae) & 106 & 16,51 & 23 & 6,57 & 129 & 13,05 \\
\hline \multicolumn{7}{|l|}{ Platypodidae } \\
\hline Platypus linearis & 29 & 4,52 & 11 & 3,14 & 40 & 4,03 \\
\hline Platypus sp. & 11 & 1,71 & 2 & 0,57 & 13 & 1,31 \\
\hline Sub-total (Platypodidae) & 40 & 6,23 & 13 & 3,71 & 53 & 5,34 \\
\hline \multicolumn{7}{|l|}{ Scolytidae } \\
\hline Coccotrypes palmarum & 2 & 0,31 & 1 & 0,29 & 3 & 0,30 \\
\hline Coccotrypes sp. & - & - & 3 & 0,86 & 3 & 0,30 \\
\hline Corthylus convexicauda & 1 & 0,16 & - & - & 1 & 0,11 \\
\hline Cryptocarenus diadematus & 20 & 3,12 & 18 & 5,14 & 38 & 3,83 \\
\hline Cryptocarenus heveae & 8 & 1,25 & 18 & 5,14 & 26 & 2,62 \\
\hline Cryptocarenus seriatus & 14 & 2,18 & 10 & 2,86 & 24 & 2,42 \\
\hline Cryptocarenus sp. & 2 & 0,31 & 6 & 1,71 & 8 & 0,81 \\
\hline Hypothenemus bolivianus & 3 & 0,47 & - & - & 3 & 0,30 \\
\hline Hypothenemus eruditus & 30 & 4,67 & 20 & 5,71 & 50 & 5,04 \\
\hline Hypothenemus obscurus & 18 & 2,80 & 23 & 6,57 & 41 & 4,12 \\
\hline Premnobius cavipennis & 116 & 18,07 & 64 & 18,29 & 180 & 18,15 \\
\hline Sampsonius dampfi & 12 & 1,87 & 5 & 1,43 & 17 & 1,71 \\
\hline Xyleborus affinis & 115 & 17,91 & 79 & 22,57 & 194 & 19,56 \\
\hline Xyleborus compactus & - & - & 2 & 0,57 & 2 & 0,20 \\
\hline Xyleborus ferrugineus & 57 & 8,88 & 42 & 12,00 & 99 & 9,97 \\
\hline Xyleborus hagedorni & 1 & 0,16 & - & - & 1 & 0,10 \\
\hline Xyleborus neivai & - & - & 1 & 0,29 & 1 & 0,10 \\
\hline Xyleborus retusus & 29 & 4,52 & 9 & 2,57 & 38 & 3,83 \\
\hline Xyleborus sp. & 2 & 0,31 & 3 & 0,86 & 5 & 0,50 \\
\hline Xyleborus spinulosus & 66 & 10,28 & 4 & 1,14 & 70 & 7,02 \\
\hline Xyleborus squamulatus & - & - & 2 & 0,57 & 2 & 0,20 \\
\hline Tricolus sp. & - & - & 4 & 1,14 & 4 & 0,40 \\
\hline Sub-total (Scolytidae) & 496 & 77,26 & 314 & 89,71 & 810 & 81,59 \\
\hline Total & 642 & 100 & 350 & 100 & 992 & 100 \\
\hline
\end{tabular}

As espécies $P$. cavipennis, $X$. affinis e $X$. ferrugineus ocorreram como dominantes, muito abundantes, muito freqüentes e constantes, respectivamente, independentemente do período analisado, enquanto as espécies, $B$. uncinata e $X$. spinulosus ocorreram respectivamente como dominantes, muito abundantes, muito 
freqüentes e constantes somente no período seco (Tabela 3).

Morales et al. (2000) observaram em plantios de Eucalyptus grandis, no estado de Minas Gerais, que Xyleborus paraguayensis e $X$. affinis foram muito abundantes, que $X$. ferrugineus ocorreu como pouco abundante atribuindo esta diferença à baixa disponibilidade de fonte de alimento e à alta especificidade quanto a seleção de hospedeiros.

Muller \& Andreiv (2004) observaram em povoamentos de E. grandis que das 21 espécies de Scolytidae coletadas, 28,6\%, $23,8 \%$ e $47,6 \%$ ocorreram como constantes, acessórias e acidentais respectivamente.

Segundo Clemente (1995) a ocorrência de alta percentagem de espécies acidentais em comunidades florestais pode ser indicativo de resistência do meio à proliferação das espécies, enquanto que as espécies classificadas como muito abundantes, constantes e dominantes indicariam o estabelecimento de comunidades.

No talhão de urograndis ocorreu índice de equitatibilidade de 0,82 no período de seca, indicando condições ambientais favoráveis para densidades populacionais mais homogêneas das espécies associadas ao ambiente. $\mathrm{O}$ índice de Margalef mostrou que no período de chuva ocorreu maior riqueza de espécies, mas com densidades populacionais heterogêneas (Tabela 4), enquanto que na análise anual de ShanonWiener ficou evidente que o período anual é diversificado em quantidades de espécies com populações mais homogêneas. Portanto, pode-se afirmar que as espécies que ocorrem neste ambiente encontram-se adaptadas às variações dos fatores climáticos da região e suas densidades populacionais são reguladas de acordo com as condições ambientais locais.

As espécies $X$. ferrugineus e $X$. affinis ocorreram com picos populacionais em setembro e com baixa densidade populacional nos demais meses no talhão de urograndis. $P$. cavipennis exibiu alta abundância em abril e junho, com picos populacionais em julho e setembro, enquanto $X$. spinulosus mostrou alta densidade em julho, com sucessivo decréscimo nos demais meses do ano (Figura 1).

Peres Filho et al. (2005) observaram que $P$. cavipennis apresentou picos populacionais no inicio e final do período de seca. Isto confirma a ocorrência de surtos populacionais em talhões de eucalipto nesta época do ano. Este resultado pode ser atribuído ao déficit hídrico ocasionado pelo período de estiagem prolongado na região, que pode ter causado estresse e debilitado as defesas físicas e químicas das árvores, tornando-as susceptíveis à colonização de coleobrocas.

Nesta região, nos meses de seca, é frequente a mortalidade de árvores, independente da idade, corroborando as informações obtidas para $P$. cavipennis por Trefflich (2003), que observou o mesmo resultado em talhões do híbrido de E. grandis x E. urophylla, no município de Brotas-SP. Os picos populacionais de $P$. cavipennis ocorreram durante os meses de março a Junho, período em que a precipitação na região foi quase nula, indicando que independente da região, a espécie de coleobroca tem seu ritmo de atividade associado ao fator abiótico.

No talhão de urograndis, a temperatura máxima e umidade relativa foram os fatores climáticos com os maiores valores e quantidades de correlações significativas, mostrando que as densidades populacionais de Xyleborus affinis e $X$. ferrugineus podem ser afetadas pelas variações destes fatores na região. $X$. spinulosus correlacionou-se fortemente com diversos fatores climáticos, demonstrando que o ritmo de algumas atividades desta espécie, como o vôo de dispersão, de acasalamento, de seleção e colonização de novos hospedeiros, podem ser influenciados 
Tabela 3. Índices faunísticos das espécies de coleópteros coletadas com armadilhas etanólicas, nos períodos de seca e de chuva no talhão de urograndis (Eucalyptus urophylla $\mathrm{x}$ Eucalyptus grandis), município de Cuiabá-MT.

\begin{tabular}{|c|c|c|c|c|c|c|c|c|c|c|c|c|}
\hline \multirow{2}{*}{ Espécies } & \multicolumn{4}{|c|}{ Seca } & \multicolumn{4}{|c|}{ Chuva } & \multicolumn{4}{|c|}{ Anual } \\
\hline & $\mathrm{D}$ & A & $\mathrm{F}$ & $\mathrm{C}$ & $\mathrm{D}$ & $\mathrm{A}$ & $\mathrm{F}$ & $\mathrm{C}$ & $\mathrm{D}$ & $\mathrm{A}$ & $\mathrm{F}$ & $\mathrm{C}$ \\
\hline Bostrychopsis uncinata & $\mathrm{d}$ & $\mathrm{ma}$ & $\mathrm{mf}$ & $\mathrm{W}$ & nd & $\mathrm{d}$ & $\mathrm{pf}$ & $\mathrm{y}$ & $\mathrm{d}$ & $\mathrm{a}$ & $\mathrm{mf}$ & $\mathrm{y}$ \\
\hline Micrapate sp. & d & $\mathrm{c}$ & $\mathrm{f}$ & $\mathrm{w}$ & nd & d & $\mathrm{pf}$ & $\mathrm{y}$ & $\mathrm{d}$ & $\mathrm{c}$ & $\mathrm{f}$ & $\mathrm{W}$ \\
\hline Xyloperthela picea & nd & $\mathrm{c}$ & $\mathrm{f}$ & $\mathrm{W}$ & nd & $r$ & $\mathrm{pf}$ & $\mathrm{y}$ & nd & $\mathrm{c}$ & $\mathrm{f}$ & $\mathrm{W}$ \\
\hline Xyloprista sp. & nd & $\mathrm{r}$ & $\mathrm{pf}$ & $\mathrm{y}$ & nd & $\mathrm{c}$ & $\mathrm{f}$ & $\mathrm{W}$ & nd & $\mathrm{d}$ & $\mathrm{pf}$ & $\mathrm{W}$ \\
\hline Platypus linearis & $\mathrm{d}$ & $\mathrm{c}$ & $\mathrm{f}$ & $\mathrm{W}$ & nd & $\mathrm{c}$ & $\mathrm{f}$ & W & $\mathrm{d}$ & $\mathrm{c}$ & $\mathrm{f}$ & W \\
\hline Platypus sp. & nd & d & $\mathrm{pf}$ & $\mathrm{w}$ & nd & $\mathrm{r}$ & $\mathrm{pf}$ & $\mathrm{y}$ & nd & $\mathrm{d}$ & $\mathrm{pf}$ & $\mathrm{W}$ \\
\hline Coccotrypes palmarum & nd & $\mathrm{r}$ & $\mathrm{pf}$ & $\mathrm{Z}$ & nd & $\mathrm{r}$ & $\mathrm{pf}$ & $\mathrm{Z}$ & nd & $\mathrm{r}$ & $\mathrm{pf}$ & $\mathrm{z}$ \\
\hline Coccotrypes sp. & - & - & - & - & nd & $\mathrm{r}$ & $\mathrm{pf}$ & $\mathrm{y}$ & nd & $\mathrm{r}$ & $\mathrm{pf}$ & $\mathrm{z}$ \\
\hline Corthylus convexicauda & nd & $\mathrm{r}$ & $\mathrm{pf}$ & $\mathrm{Z}$ & - & - & - & - & nd & $\mathrm{r}$ & pf & $\mathrm{z}$ \\
\hline Cryptocarenus diadematus & nd & $\mathrm{c}$ & $\mathrm{f}$ & $\mathrm{W}$ & d & $\mathrm{c}$ & $\mathrm{f}$ & $\mathrm{W}$ & $\mathrm{d}$ & $\mathrm{c}$ & $\mathrm{f}$ & $\mathrm{W}$ \\
\hline Cryptocarenus heveae & nd & $\mathrm{r}$ & $\mathrm{pf}$ & $\mathrm{w}$ & d & $\mathrm{c}$ & $\mathrm{f}$ & $\mathrm{W}$ & nd & $\mathrm{c}$ & $\mathrm{f}$ & $\mathrm{W}$ \\
\hline Cryptocarenus seriatus & nd & d & $\mathrm{pf}$ & $\mathrm{w}$ & nd & $\mathrm{c}$ & $\mathrm{f}$ & $\mathrm{w}$ & nd & $\mathrm{c}$ & $\mathrm{f}$ & $\mathrm{W}$ \\
\hline Cryptocarenus sp. & nd & $\mathrm{r}$ & $\mathrm{pf}$ & $\mathrm{z}$ & nd & $\mathrm{d}$ & $\mathrm{pf}$ & $\mathrm{Z}$ & nd & $\mathrm{r}$ & $\mathrm{pf}$ & $\mathrm{z}$ \\
\hline Hypothenemus bolivianus & nd & $\mathrm{r}$ & $\mathrm{pf}$ & $\mathrm{w}$ & - & - & - & - & nd & $\mathrm{r}$ & $\mathrm{pf}$ & $\mathrm{y}$ \\
\hline Hypothenemus eruditus & $\mathrm{d}$ & $\mathrm{c}$ & $\mathrm{f}$ & $\mathrm{w}$ & d & $\mathrm{c}$ & $\mathrm{f}$ & $\mathrm{W}$ & $\mathrm{d}$ & $\mathrm{c}$ & $\mathrm{f}$ & $\mathrm{W}$ \\
\hline Hypothenemus obscurus & nd & $\mathrm{c}$ & $\mathrm{f}$ & $\mathrm{w}$ & d & $\mathrm{a}$ & $\mathrm{mf}$ & $\mathrm{W}$ & $\mathrm{d}$ & $\mathrm{c}$ & $\mathrm{f}$ & $\mathrm{W}$ \\
\hline Premnobius cavipennis & $\mathrm{d}$ & $\mathrm{ma}$ & $\mathrm{mf}$ & $\mathrm{w}$ & d & $\mathrm{ma}$ & $\mathrm{mf}$ & w & $\mathrm{d}$ & $\mathrm{m}$ & $\mathrm{mf}$ & $\mathrm{W}$ \\
\hline Sampsonius dampfi & nd & $\mathrm{d}$ & $\mathrm{pf}$ & $\mathrm{w}$ & nd & $\mathrm{d}$ & $\mathrm{pf}$ & $\mathrm{y}$ & nd & $\mathrm{d}$ & $\mathrm{pf}$ & $\mathrm{W}$ \\
\hline Xyleborus affinis & $\mathrm{d}$ & $\mathrm{ma}$ & $\mathrm{mf}$ & $\mathrm{w}$ & d & $\mathrm{ma}$ & $\mathrm{mf}$ & $\mathrm{W}$ & $\mathrm{d}$ & $\mathrm{m}$ & $\mathrm{mf}$ & $\mathrm{W}$ \\
\hline Xyleborus compactus & - & - & - & - & nd & $\mathrm{r}$ & $\mathrm{pf}$ & $\mathrm{y}$ & nd & $\mathrm{r}$ & $\mathrm{pf}$ & $\mathrm{z}$ \\
\hline Xyleborus ferrugineus & $\mathrm{d}$ & $\mathrm{ma}$ & $\mathrm{mf}$ & $\mathrm{w}$ & d & $\mathrm{ma}$ & $\mathrm{mf}$ & $\mathrm{W}$ & $\mathrm{d}$ & $\mathrm{m}$ & $\mathrm{mf}$ & $\mathrm{W}$ \\
\hline Xyleborus hagedorni & nd & $\mathrm{r}$ & $\mathrm{pf}$ & $\mathrm{Z}$ & - & - & - & - & nd & $\mathrm{r}$ & $\mathrm{pf}$ & $\mathrm{Z}$ \\
\hline Xyleborus neivai & - & - & - & - & nd & $\mathrm{r}$ & $\mathrm{pf}$ & $\mathrm{Z}$ & nd & $\mathrm{r}$ & $\mathrm{pf}$ & $\mathrm{Z}$ \\
\hline Xyleborus retusus & $\mathrm{d}$ & $\mathrm{c}$ & $\mathrm{f}$ & $\mathrm{W}$ & nd & $\mathrm{c}$ & $\mathrm{f}$ & $\mathrm{W}$ & $\mathrm{d}$ & $\mathrm{c}$ & $\mathrm{f}$ & W \\
\hline Xyleborus sp. & nd & $\mathrm{r}$ & $\mathrm{pf}$ & $\mathrm{z}$ & nd & $\mathrm{r}$ & $\mathrm{pf}$ & $\mathrm{Z}$ & nd & $\mathrm{r}$ & $\mathrm{pf}$ & $\mathrm{Z}$ \\
\hline Xyleborus spinulosus & $\mathrm{d}$ & $\mathrm{ma}$ & $\mathrm{mf}$ & $\mathrm{w}$ & nd & $\mathrm{d}$ & $\mathrm{pf}$ & $\mathrm{w}$ & $\mathrm{d}$ & $\mathrm{m}$ & $\mathrm{mf}$ & $\mathrm{W}$ \\
\hline Xyleborus squamulatus & - & - & - & - & nd & $r$ & $\mathrm{pf}$ & $\mathrm{Z}$ & nd & $\mathrm{r}$ & $\mathrm{pf}$ & $\mathrm{Z}$ \\
\hline Tricolus sp. & - & - & - & - & nd & $\mathrm{d}$ & $\mathrm{pf}$ & $\mathrm{y}$ & nd & $\mathrm{r}$ & $\mathrm{pf}$ & $\mathrm{Z}$ \\
\hline
\end{tabular}

D: Dominância - (sd) super dominante; (d) dominante; (nd) não dominante. A: Abundância - (sa) super abundante; (ma) muito abundante; (a) abundante; (c) comum; (d) dispersa; (r) rara. F: Frequência - (sf) super frequente; (mf) muito frequente; (f) frequente; (pf) pouco frequente. C: Constância - (w) constante; (y) acessória; (z) acidental.

Tabela 4. Índices de diversidade para os períodos de seca, chuva para o talhão de urograndis (Eucalyptus urophylla x Eucalyptus grandis), município de Cuiabá-MT.

\begin{tabular}{ccccccccc}
\hline \multicolumn{2}{c}{ Equitabilidade $(\mathrm{E})$} & \multicolumn{3}{c}{ Margalef $(\alpha)$} & \multicolumn{3}{c}{ Shannon-Wiener $(\mathrm{H})$} \\
\hline Seca & Chuva & Anual & Seca & Chuva & Anual & Seca & Chuva & Anual \\
\hline 0,8185 & 0,7919 & 0,7907 & 3,4032 & 4,0970 & 3,9132 & 2,5665 & 2,5491 & 2,6346 \\
\hline
\end{tabular}

$\mathrm{E}=\{0 ; 1\}$. Variância $\mathrm{H}_{\mathrm{SECA}}=0,0012$; Variância $\mathrm{H}_{\mathrm{CHUVA}}=0,0031$; Variância $\mathrm{H}_{\mathrm{ANUAL}}=0,0010$. 

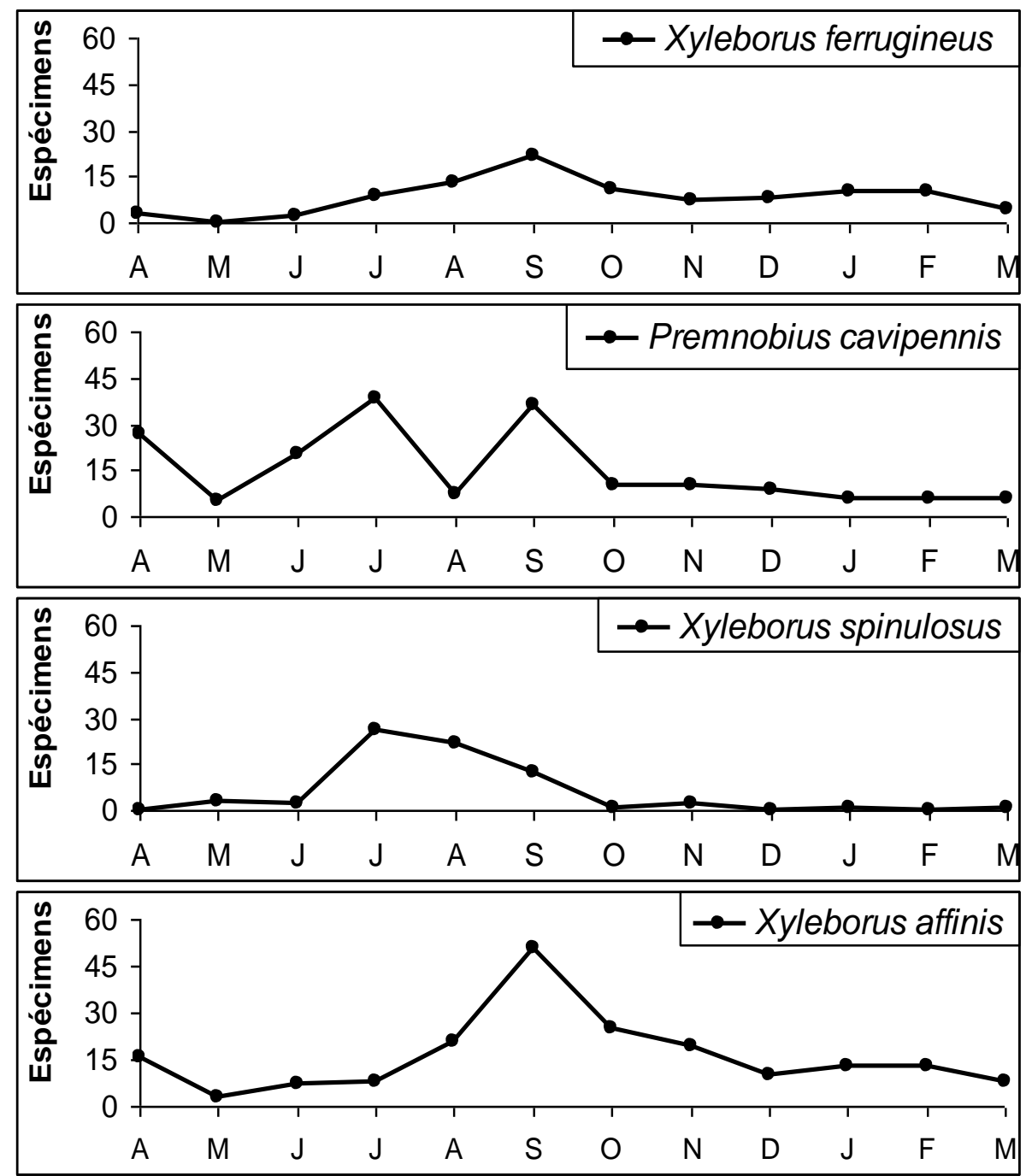

Figura 1. Flutuação populacional para as espécies selecionadas no talhão de urograndis (Eucalyptus urophylla x Eucalyptus grandis), município de Cuiabá-MT.

Tabela 5. Correlação entre os fatores temperatura máxima, média e mínima, umidade relativa e precipitação pluvial e as espécies de Scolytidae coletados com armadilhas etanólicas no talhão de urograndis (Eucalyptus urophylla x Eucalyptus grandis), município de Cuiabá-MT.

\begin{tabular}{lccccc}
\hline \multirow{2}{*}{ Espécies } & \multicolumn{3}{c}{ Temperatura $\left({ }^{\circ} \mathrm{C}\right)$} & Umidade & Precipitação \\
\cline { 2 - 6 } & Máxima & Mínima & Média & Relativa $(\%)$ & Pluvial \\
\hline Premnobius cavipennis & $0,22^{*}$ & $-0,62^{*}$ & $-0,38^{*}$ & $-0,16^{*}$ & $-0,23^{*}$ \\
Xyleborus affinis & $0,60^{*}$ & $-0,16^{*}$ & $0,31^{*}$ & $-0,57^{*}$ & $-0,06^{*}$ \\
Xyleborus ferrugineus & $0,71^{*}$ & $-0,20^{*}$ & $0,35^{*}$ & $-0,77^{*}$ & $-0,20^{*}$ \\
Xyleborus spinulosus & $0,62^{*}$ & $-0,80^{*}$ & $-0,24^{*}$ & $-0,67^{*}$ & $-0,66^{*}$ \\
\hline
\end{tabular}

*Significativo ao nível de 5\% de probabilidade. 
Dinâmica populacional de Bostrichidae, Platypodidae e Scolytidae (Coleoptera) em talhão de urograndis (Eucalyptus urophylla $x$ Eucalyptus grandis) no município de Cuiabá, estado de Mato Grosso

Tabela 6. Análise de variância das espécies de coleópteros coletados no talhão de urograndis (Eucalyptus urophylla x Eucalyptus grandis), município de Cuiabá-MT.

\begin{tabular}{lccccc}
\hline Fontes de Variação & G.L. & S.Q. & Q.M. & Fc. & Signif. \\
\hline Períodos & 1 & 1,2740 & 1,2740 & $29,59 * *$ & 0,00000 \\
Espécies & 27 & 35,5555 & 1,3169 & $30,59^{* *}$ & 0,00000 \\
Períodos x Espécies & 27 & 6,0591 & 0,2244 & $5,21 * *$ & 0,00000 \\
Resíduo & 280 & 12,0546 & 0,0431 & & \\
\hline Total & 335 & 54,9432 & & & \\
\hline Transf.: $\log (\mathrm{x}+1) ;$ C.V.(\%) $=54.959$ & & &
\end{tabular}

Tabela 7. Valores médios para espécies de coleópteros coletados no talhão de urograndis (Eucalyptus urophylla x Eucalyptus grandis), município de Cuiabá-MT.

\begin{tabular}{|c|c|c|c|c|c|c|}
\hline \multirow{3}{*}{$\begin{array}{c}\text { Espécies } \\
\text { Bostrychopsis uncinata }\end{array}$} & \multicolumn{6}{|c|}{ Médias } \\
\hline & \multicolumn{2}{|c|}{ Seca } & \multicolumn{2}{|c|}{ Chuva } & \multicolumn{2}{|c|}{ Anual } \\
\hline & 0,8794 & $\mathrm{Ba}$ & 0,2300 & $\mathrm{Db}$ & 0,5547 & $\mathrm{C}$ \\
\hline Micrapate sp. & 0,7823 & $\mathrm{Ca}$ & 0,2300 & $\mathrm{Db}$ & 0,5062 & $\mathrm{C}$ \\
\hline Xyloperthela picea & 0,5557 & $\mathrm{Da}$ & 0,1003 & $\mathrm{~Eb}$ & 0,3280 & $\mathrm{D}$ \\
\hline Xyloprista sp. & 0,2594 & $\mathrm{Ea}$ & 0,3674 & $\mathrm{Da}$ & 0,3134 & $\mathrm{D}$ \\
\hline Platypus linearis & 0,6834 & $\mathrm{Ca}$ & 0,4392 & $\mathrm{Db}$ & 0,5613 & $\mathrm{C}$ \\
\hline Platypus sp. & 0,3465 & $\mathrm{Ea}$ & 0,1003 & $\mathrm{~Eb}$ & 0,2234 & $\mathrm{D}$ \\
\hline Coccotrypes palmarum & 0,0795 & $\mathrm{Fa}$ & 0,0502 & $\mathrm{Ea}$ & 0,0648 & $\mathrm{E}$ \\
\hline Coccotrypes sp. & - & - & 0,1505 & $\mathrm{E}$ & 0,0753 & $\mathrm{E}$ \\
\hline Corthylus convexicauda & 0,0502 & $\mathrm{~F}$ & - & - & 0,0251 & $\mathrm{E}$ \\
\hline Cryptocarenus diadematus & 0,4798 & $\mathrm{Da}$ & 0,5102 & $\mathrm{Ca}$ & 0,4950 & $\mathrm{C}$ \\
\hline Cryptocarenus heveae & 0,3304 & $\mathrm{~Eb}$ & 0,5766 & $\mathrm{Ca}$ & 0,4535 & $\mathrm{C}$ \\
\hline Cryptocarenus seriatus & 0,5102 & $\mathrm{Da}$ & 0,3415 & $\mathrm{Da}$ & 0,4259 & $\mathrm{C}$ \\
\hline Cryptocarenus sp. & 0,1003 & $\mathrm{Fa}$ & 0,2509 & $\mathrm{Da}$ & 0,1756 & $\mathrm{D}$ \\
\hline Hypothenemus bolivianus & 0,1505 & $\mathrm{~F}$ & - & - & 0,0753 & $\mathrm{E}$ \\
\hline Hypothenemus eruditus & 0,7085 & $\mathrm{Ca}$ & 0,6191 & $\mathrm{Ca}$ & 0,6638 & $\mathrm{C}$ \\
\hline Hypothenemus obscurus & 0,5340 & $\mathrm{Da}$ & 0,6476 & $\mathrm{Ca}$ & 0,5908 & $\mathrm{C}$ \\
\hline Premnobius cavipennis & 1,2585 & $\mathrm{Aa}$ & 1,0346 & $\mathrm{Aa}$ & 1,1466 & A \\
\hline Sampsonius dampfi & 0,3920 & $\mathrm{Ea}$ & 0,2300 & $\mathrm{Da}$ & 0,3110 & $\mathrm{D}$ \\
\hline Xyleborus affinis & 1,2831 & $\mathrm{Aa}$ & 1,1338 & $\mathrm{Aa}$ & 1,2084 & A \\
\hline Xyleborus compactus & - & - & 0,1003 & $\mathrm{E}$ & 0,0502 & $\mathrm{E}$ \\
\hline Xyleborus ferrugineus & 0,9721 & $\mathrm{Ba}$ & 0,8395 & $\mathrm{Ba}$ & 0,9058 & $\mathrm{~B}$ \\
\hline Xyleborus hagedorni & 0,0502 & $\mathrm{~F}$ & - & - & 0,0251 & $\mathrm{E}$ \\
\hline Xyleborus neivai & - & - & 0,0502 & $\mathrm{E}$ & 0,0251 & $\mathrm{E}$ \\
\hline Xyleborus retusus & 0,7309 & $\mathrm{Ca}$ & 0,3257 & $\mathrm{Db}$ & 0,5283 & $\mathrm{C}$ \\
\hline Xyleborus sp. & 0,1003 & $\mathrm{Fa}$ & 0,1297 & $\mathrm{Ea}$ & 0,1150 & $\mathrm{E}$ \\
\hline Xyleborus spinulosus & 1,0576 & $\mathrm{Ba}$ & 0,1297 & $\mathrm{~Eb}$ & 0,5937 & $\mathrm{C}$ \\
\hline Xyleborus squamulatus & - & - & 0,0795 & $\mathrm{E}$ & 0,0398 & $\mathrm{E}$ \\
\hline Tricolus sp. & - & - & 0,1799 & $\mathrm{E}$ & 0,0899 & $\mathrm{E}$ \\
\hline Médias de Períodos & 0,4391 & $\mathrm{~A}$ & 0,3160 & $\mathrm{~b}$ & & \\
\hline
\end{tabular}


pelas variações dos fatores climáticos nesta região (Tabela 5). Dorval (2002) observou em talhão de $E$. pellita, que $X$. ferrugineus tem relação negativa com a umidade relativa, enquanto Dall'oglio \& Peres Filho (1997) observaram que a espécie apresenta correlação positiva com a temperatura média em plantios de $H$. brasiliensis, no município de Itiquira, estado de Mato Grosso.

A análise de variância para a abundância das espécies ocorridas no talhão de urograndis revelou diferenças estatísticas significativas ao nível de $1 \%$ de probabilidade para todas as fontes de variação analisadas (Tabela 6).

A comparação entre médias das espécies coletadas no talhão do clone urograndis indica que $X$. affinis, e $P$. cavipennis exibem valores médios maiores de indivíduos coletados diferenciando-se significativamente das demais espécies, independente do período analisado (Tabela 7). Dentre as 28 espécies estudadas, oito apresentaram diferenças significativas considerando os períodos de seca e de chuva.

Confirmando os resultados obtidos nesta pesquisa, Morales et al. (2000), pesquisando a ocorrência de escolitídeos em plantios de E. grandis em Minas Gerais, relataram que Xyleborus paraguayensis e $X$. affinis, totalizaram 26.981 indivíduos capturados nos talhões amostrados.

Nos estudos desenvolvidos por Trefflich (2003), constataram-se diferenças estatísticas significativas entre as médias de $X$. affinis, e $P$. cavipennis, sendo elas as espécies mais coletadas nos quatro talhões amostrados.

Aplicando o teste de média de Kruskal-Wallis, foi verificado que $P$. cavipennis foi a espécie mais frequiente independentemente do Incremento Médio Anual (IMA) dos talhões amostrados, seguida das espécies $H$. obscurus e $C$. heveae, que foram mais abundantes em talhões de IMA intermediários.
Zanuncio et al. (2005) registraram ataques de $P$. cavipennis e Premnobius ambitiosus, Dryocoetoides cristatus e Euplatypus parallelus em Minas Gerais em plantios com dois anos de idade e com diâmetro médio de $6,5 \mathrm{~cm}$ à altura do peito, com danos concentrando-se no intervalo de 0,5 a $1,5 \mathrm{~m}$ de altura.

\section{CONCLUSÃO}

O plantio clonal de urograndis é um ambiente favorável para a ocorrência de altas densidades populacionais de Premnobius cavipennis, Xyleborus affinis e Xyleborus ferrugineus, consideradas pragas de eucalipto no Brasil;

No talhão de urograndis, os meses de seca são períodos de maior atividade para as espécies de escolitídeos consideradas nocivas ao eucalipto na região.

\section{REFERÊNCIAS BIBLIOGRÁFICAS}

ABREU, R. L. S.; SALES-CAMPOS, C.; HANADA, R. E.; VASCONCELOS, F. J.; FREITAS, J. A. 2002. Avaliação de danos por insetos em toras estocadas em indústrias madeireiras de Manaus, Amazonas, Brasil. Sociedade de Investigações Florestais. Revista Árvore. Viçosa-MG, v. 26, n. 6, p. 789796.

BERTI FILHO, E. 1979. Coleópteros de importância florestal: 1- Scolytidae. IPEF, Piracicaba, v.19, p. 39-43.

BERTI FILHO, E. 1981. Insetos associados às plantações do gênero Eucalyptus nos estados da Bahia, Espírito Santo, Mato Grosso do Sul, Minas Gerais e São Paulo. 1981. 176p. Tese de Livre Docência, ESALQ/USP, Piracicaba.

CAMPOS, H. 1976. Estatística experimental não-paramétrica. Piracicaba, EDUSP, 322 p.

CONCEIÇÃO, P.N. (Coordenador). 1997. Manejo de bacia hidrogáfica do rio 
Coxipó-Açú para conservação de seus recursos hídricos. Brasília: ABEAS/ MMA/ SRH/UFMT, 127p.

COSTA NETO, P.L.O. 1977. Estatística. São Paulo, Edgard Blücher, 168 p.

CLEMENTE, A. T.C. 1995. Análise de populações de Lepidoptera em comunidades florestais de Araucaria angustifolia, Eucalyptus grandis e Pinus taeda. Curitiba, 1995. 75 f. Dissertação (Mestrado em Ciências Florestais). Universidade Federal do Paraná.

DALL'OGLIO, O. T.; PERES FILHO. O. 1997. Levantamento e flutuação de populacional de coleobrocas em plantios homogêneos de seringueira em ItiquiraMT. Revista Scientia Forestalis, Piracicaba, v.51, n. 2, p.49-58.

DORVAL, A. 2002. Levantamento populacional de coleópteros com armadilhas etanólicas em plantios de eucaliptos e em uma área com vegetação de cerrado no município de Cuiabá, Estado de Mato Grosso. 2002. 143p. Tese (Doutorado em Ciências Biológicas). Universidade Federal do Paraná. Curitiba.

DORVAL, A.; PERES FILHO O.; MARQUES E. N. 2004. Levantamento de Scolytidae (Coleoptera) em plantações de Eucalyptus spp. em Cuiabá, estado de Mato Grosso. Revista Ciência Florestal. Santa Maria-RS, v. 14, n. 1, p. 47-58.

LIU, L.: SCHÖNITZER, K.; YANG, J. 2008. A review of the literature on the life history of Bostrichidae. Mitt. Munch. Ent. Ges. v. 98, p. 91-97.

MATOSKI, S. L. S. Comportamento de Dinoderus minutus Fabricius (1775) (Coleoptera: Bostrichidae) em lâminas torneadas de madeira. 2005, 94p. (Mestrado em Engenharia Florestal). Universidade Federal do Paraná. Curitiba.

MORAES, R. C. B. ; SILVEIRA NETO, S. 2003.Software para análise faunística.
In: Simpósio de Controle Biológico, 8, São Paulo, Resumos, Piracicaba-SP. ESALQ. p. 95.

MORALES N. E.; ZANUNCIO J. C.; PRATISSOLI D.; FABRES A. S. 2000. Fluctuación poblacional de Scolytidae (Coleoptera) en zonas reforestadas com Eucalyptus grandis (Myrtaceae) en Minas Gerais, Brasil. Revista de Biologia Tropical. San José, Costa Rica, v. 48, n. 1, p. 129-134.

MULLER, J. A.; ANDREIV, J. 2004.Caracterização da família Scolytidae (Insecta: Coleoptera) em três ambientes florestais. Revista Cerne, Lavras-MG, v. 10, n. 1, p. 39-45.

OLIVEIRA H. G.; ZANUNCIO T. V.; ZANUNCIO J. C.; SANTOS G. P. 2001. Coleópteros associados à eucaliptocultura na região de Nova Era, Minas Gerais, Brasil. Floresta e Ambiente. Rio de Janeiro-RJ, v. 8, n. 1, p. 52-60.

PEDROSA MACEDO, J. H. 1993. Manual de pragas em florestas: pragas florestais no sul do Brasil. Viçosa: SIF / IPEF, 112p.

PERES-FILHO, O.; DORVAL A.; SIQUEIRA S. A.; BERTI-FILHO E. 2005. Levantamento de coleópteros em plantios de Eucalyptus spp. em Rondonópolis, estado de Mato Grosso. Revista de Agricultura. Piracicaba-SP v. 80, n. 2, p. 213-227.

PERES-FILHO, O.; SANTOS, A. K. G.; DORVAL, A.; BERTI-FILHO, E.; MOURA, R. G. 2006. Coleópteros em madeira estocada em pátio de serraria. Revista de Agricultura. Piracicaba-SP, v. 81, n. 2, p. 213-228.

PERES FILHO, O.; TEIXEIRA, E. P.; BEZERRA, M. L. M.; DORVAL, A.; BERTI-FILHO, E. 2006. First Record of Sinoxylon conigerum Gerstäcker (Coleoptera: Bostrichidae) in Brazil. Neotropical Entomology. v. 35, n. 5, p. 712-713. 
SANTOS, G. P.; ZANUNCIO J. C.; OLIVEIRA H. G.; ZANUNCIO T. V.; LACERDA M. C. 2003. Coleópteros coletados em plantação de Eucalyptus urophylla S. T. Blake (Myrtaceae) na região de Niquelandia, estado de Goiás, Brasil. Bioscience Journal. UberlândiaMG. v. 19, n. 3, p. 77-82.

TREFFLICH, K. 2003. Ocorrência e flutuação populacional de Scolytidae (Insecta: Coleoptera) em talhões do híbrido de Eucalyptus grandis W. Hill ex Maiden x Eucalyptus urophylla S. T. Blake com diferentes incrementos médios anuais em Brotas, São Paulo. 2003. 52p. Dissertação de Mestrado em Ciências Biológicas. UFPR. Curitiba.
ZANUNCIO, J. C.; BRAGANÇA, M.A.L.; LARANJEIRO, A.J.; FAGUNDES, M. 1993. Coleópteros associados à eucaliptocultura nas regiões de São Mateus e Aracruz, Espírito Santo. Revista Ceres, v. 41, n. 232, p. 584590.

ZANUNCIO, J. C.; SOSSAI, M. F.; FLECHTMANN, C. A. H.; ZANUNCIO V. Z.; GUIMARÃES, E. M.; ESPINDULA, M. C. 2005. Plants of an Eucalyptus clone damage by Scolytidae and Platypodidae (Coleoptera). Revista de Pesquisa Agropecuária Brasileira. Brasília-DF, v. 40, n. 5 , p. 513-515. 